\title{
Simplified Model of Offshore Airborne Wind Energy Converters
}

\author{
Antonello Cherubini ${ }^{\mathrm{a}}$, Rocco Vertechy ${ }^{\mathrm{b}}$, Marco Fontana ${ }^{\mathrm{a}, *}$ \\ ${ }^{a}$ Scuola Superiore Sant'Anna, Piazza dei Martiri 33, Pisa, Italy \\ ${ }^{b}$ University of Bologna, Viale Risorgimento 2, 40136 Bologna, Italy
}

\begin{abstract}
Airborne Wind Energy Converters (AWECs) are promising devices that, thanks to tethered airborne systems, are able to harvest energy of winds blowing at an altitude which is not reachable by traditional wind turbines. This paper is meant to provide an analysis and a preliminary evaluation of an AWEC installed on a floating offshore platform. A minimum complexity dynamic model is developed including a moored heaving platform coupled with the dynamics of an AWEC in steady crosswind flight. A numerical case study is presented through the analysis of different geometrical sizes for the platform and for the airborne components. The results show that offshore AWECs are theoretically viable and they may also be more efficient than grounded device by taking advantage of a small amount of additionally harvested power from ocean waves.
\end{abstract}

Keywords: floating, AWE, wave energy, high altitude wind, offshore renewable, offshore platform

\section{Introduction}

The last years have seen a dramatic growth of a new sector in renewable energy technologies which aim at the development of Airborne Wind Energy Converters (AWECs), that are a new kind of wind generators that extract energy 5 from high altitude winds by means of tethered kites or aircraft. The scientific

\footnotetext{
${ }^{*}$ Corresponding author

Email address: m.fontana@sssup.it (Marco Fontana)
} 
community and the Industry are increasingly focusing their attention on this technology because of its potential to provide low-cost renewable energy [1]. The economics of AWECs is very promising for mainly two reasons. First, winds high above ground level are steadier and typically much more powerful, persistent and globally available than those closer to the ground [2], and second, the structure of AWECs is expected to be orders of magnitude lighter (and thus cheaper) than conventional wind turbines [3].

On the downside, a possible limitation for the global development of AWEC could be the availability of significant land and air spaces required during operation. There are several strategies that are proposed to overcome this issue: (1) for land surfaces, an improvement could be obtained by allowing airborne system to fly over living or industrial areas but this would raise important Not-In-MyBackYard (NIMBY) issues; (2) optimized use of airspace could be obtained through farm installations where multiple devices share the same volume of air 20 [4].

Recently, there has been an increasing focus on bringing wind turbines offshore because, when compared to conventional onshore systems, they can rely on more powerful winds and they exploit cheaper offshore 'land'. However, offshore wind farms are expensive because their installation requires costly foundations 25 and maintenance. Today, in Europe, the offshore installed capacity accounts for 6.6 GW out of a total 117.3 GW [5]. All the offshore wind farms are fixed to the seabed in shallow water at depths usually lower than 20 meters [6]. However, the global interest is set on offshore deep water floating installations where water depth reaches several hundreds meters, due to the huge availability of sites 30 [7]. A few experimental full scale floating wind turbines have been deployed but unfortunately, they are expensive and require large submerged foundations, for example the Hywind turbine has a submerged structure 100 meters deep with a water mass displacement (hereafter simply referred to as 'displacement') around 5300 tons [8].

35 Since floating AWECs may take advantage of both the lightweight design of AWECs and the huge availability of low-cost sites for the installation of floating 
structures, this work presents a preliminary investigation on the feasibility and on first design issues of offshore AWECs.

Section 2 is an introduction to modelling offshore AWECs. Section 3 intro-

40 duces a simple dynamic model for an offshore pumping AWEC with catenary mooring. In section 4 , a case study is analysed in order to address first design issues and to estimate the advantage that an offshore AWEC could obtain by exploiting the available wave energy in addition to that of wind.

\section{Offshore AWECs}

The study and development of offshore AWECs combine different fields of engineering. They are composed of a flying wing (or kite) linked with a tether to a floating platform, which in turn is anchored to the seabed by a mooring system as shown in Fig. 1. All these subsystems involve complex dynamics and can be studied with different degrees of accuracy.

50 Depending on where the generators are placed, two types of AWECs can be envisioned:

- 'Float-gen' (floating equivalent of fround-gen) in case the generators are placed on the floating platform.

- 'Fly-gen' in case the generators are placed on board the wing.

In float-gen systems, the generation type is traction based and the aircraft performs the pumping cycle. Electricity is generated during the reel-out phase of the cycle when the aircraft generates significant pull and the cables are reeled out from the drums on which they are wound. Then comes the reel-in phase in which the aircraft is controlled in order to generate less tension and the cables are reeled back in. Reeling-in of cables is achieved with the aircraft in a depowered configuration. For current experimental systems, the reel-in phase requires nearly one third of the power produced during the reel-out phase $[9,10]$ but there are several concepts that aim at reducing substantially this power requirement [11]. 


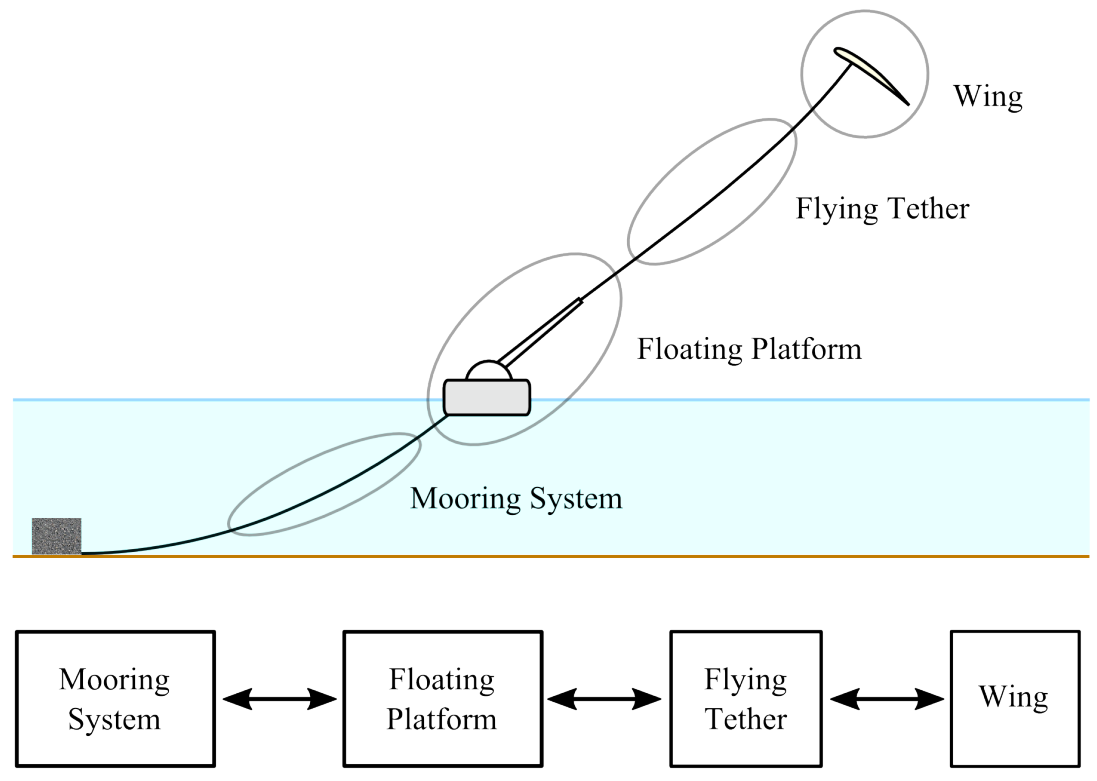

Figure 1: Schematic layout of an offshore Airborne Wind Energy Converter - The four subsystems composing an offshore AWEC are shown, i.e. wing, tether, floating platform and mooring system. The forces transmitted among the chain of components are indicated in the block diagram. 
On the other hand, fly-gen systems extract electricity from on board wind turbines which rotate fast and continuously. With respect to float-gen systems, they can have higher global electrical efficiencies and 100\% duty-cycle efficiency (they are not subjected to reel-out reel-in cycles). However, the transmission of electricity from the wing to the floating platform adds a lot more complexity and requires larger-sized cables, thus increasing the aerodynamic drag, which has a detrimental effect on crosswind power output [3]. In this work float-gen systems are analysed.

The aerodynamics of the AWEC can be investigated through different models; for example it can be described by a simple algebraic formula for quick power assessment [12], or can be modelled with a first order non linear dynamic system for controller design [13], or can be thoroughly simulated to investigate how a kite deforms during flight manoeuvres [14]. Also the cable dynamics can be taken into account when modelling the aircraft forces $[15,16]$.

In order to model the displacement of the floating platform and to estimate its effect on the energy production, it is necessary to investigate the hydrodynamics of the system. The hydrodynamics of floating bodies involve highly non-linear phenomena and turbulent flows. Reasonable predictions and simulations can be obtained by means of computationally intensive Computational Fluid Dynamic (CFD) analyses. However, several simplified methods are com85 monly employed in marine engineering to efficiently perform preliminary design iterations $[17,18,19]$.

The mooring system cannot be neglected when modelling an offshore AWEC, even though it is only needed to hold the generator in place. Several kinds of mooring systems are available and extensive literature, patents and regulations exist for oil drilling platforms and naval engineering [20, 21]. Mooring systems are known to be difficult to model due to their inherent non-linearity and sophisticated fluid structure interaction. For example, simple slack mooring systems have a non linear stiffness that changes significantly with the applied load and other design criteria [22]. In offshore oil platforms, their dynamics are usually deemed to be negligible for non-extreme events. However, it is important to 
notice that mooring equipment could be the most costly subsystem of a floating platform and could affect substantially the global business plan [23].

In this paper, a preliminary study of offshore AWECs is performed thanks to a simplified model with minimum complexity that allows analysis of the coupling of two main systems, namely a moored floating platform and an airborne device. This model has the important advantage of being computationally fast and easy to use. It is therefore suitable for qualitative analyses and first design iterations. In particular, the next section proposes a model of a 1 Degree of Freedom (DoF) heaving platform coupled with a steady state aerodynamic model of a generic wing flying in the crosswind direction.

The study only focuses on an AWEC in operational conditions, during energy production phase. Although relevant, other aspects and operating modes, such as launching/landing/emergency manoeuvres, optimal control, etc. are not discussed [1].

\section{Model}

This section describes the simple model shown in Fig. 1 that has been taken as reference for the numerical study provided in the following section.

\subsection{Hydrodynamic model}

115

The offshore floating platform is modelled as a heaving rigid body having only 1 DoF. This approximation, often assumed in the preliminary design phases of buoy-like Wave Energy Converters (WECs) [24], limits the capability and accuracy of the model. However, this approach is very useful to provide a first (quick) insight into the global behaviour of the floating dynamic system.

The forces acting on the system are shown in Fig. 2. Under these assumptions, the vertical equilibrium of the platform yields

$$
M \ddot{z}(t)=f_{\mathrm{h}}(t)+f_{\mathrm{g}}+f_{\mathrm{m}}(t)+f_{\mathrm{k}}(t)
$$




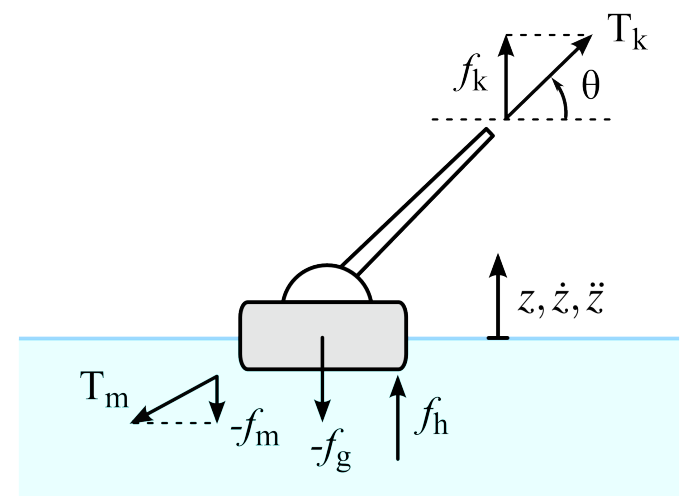

Figure 2: Model of the floating platform. - The offshore platform is modelled as a heaving floating rigid body having only 1 DoF. The horizontal components of the mooring force $T_{\mathrm{m}}$ and the aircraft force $T_{\mathrm{k}}$ are equal.

where $z$ is the heaving coordinate and $M$ is the nominal mass, i.e. the actual mass of the floating platform. On the right-hand side of the equation, there are the time-varying forces acting on the platform: $f_{\mathrm{h}}$ are the hydrodynamic forces on the hull, $f_{\mathrm{g}}$ is the gravity force, $f_{\mathrm{m}}$ and $f_{\mathrm{k}}$ are respectively the contributions of the traction forces of the mooring system and of the tethered aircraft.

\subsubsection{Platform}

The hydrodynamic force $f_{\mathrm{h}}$ represents the vertical component of the resultant of pressure and shear forces on the wet surface of the hull. It is generally calculated through an integral equation which is not easy to compute and several methods exist for its evaluation, such as CFD, analytical models or experimental measures. However, assuming inviscid and irrotational fluids subjected to small amplitude wave fields, linear wave theory can be applied to provide a reasonable estimate that is valid in non-extreme events. In particular, the hydrodynamic force on the hull can be described as the sum of three terms [25, 26]:

$$
f_{\mathrm{h}}(t)=f_{\mathrm{b}}(t)+f_{\mathrm{r}}(t)+f_{\mathrm{e}}(t)
$$


where

$$
\begin{aligned}
& f_{\mathrm{b}}(t)=-k_{\mathrm{b}} z(t)-f_{\mathrm{b} 0} \\
& f_{\mathrm{r}}(t)=-M_{\infty} \ddot{z}-\int_{0}^{t} k_{\mathrm{r}}(t-\tau) \dot{z}(\tau) \mathrm{d} \tau \\
& f_{\mathrm{e}}(t)=\sum_{\mathrm{i}=1}^{n} F_{\mathrm{wi}} \sin \left(\omega_{\mathrm{i}} t+\phi_{\mathrm{i}}\right) .
\end{aligned}
$$

In Eq. $2, f_{\mathrm{b}}$ is the buoyancy force and it is composed by a constant term $f_{\mathrm{b} 0}$ plus an elastic contribution with stiffness $k_{\mathrm{b}}$. $f_{\mathrm{r}}$ is the radiation force that takes into account the the kinetic and dissipative contributions of the motion of coordinate $z$ is made.

\subsubsection{Mooring}

The mooring system is difficult to model and its effect on the dynamics of the floating platform significantly depends on the design criteria. The mooring 155 system chosen in this analysis is composed by a single catenary line with a gravity anchor because of its simplicity and suitability for WECs [21]. Assuming, as above, linear wave theory, the catenary line dynamics are reduced to an equivalent mass, damping and stiffness, which are then added to the platform equation of motion as described in [27]. 
The expression for the mooring force is

$$
f_{\mathrm{m}}=-M_{\mathrm{m}} \ddot{z}-B_{\mathrm{m}} \dot{z}-k_{\mathrm{m}} z
$$

where $M_{\mathrm{m}}, B_{\mathrm{m}}, k_{\mathrm{m}}$ are the mooring linearised coefficients: equivalent mass, damping and stiffness respectively. $M_{\mathrm{m}}$ is not simply the nominal mooring mass, but includes also the added mass of water displaced by the mooring line and takes into account the fact that the mooring line does not move uniformly along its length.

\subsection{Aerodynamic model}

The aircraft model that is assumed in this paper is based on the work done in [28]. In addition, we have also taken into account the angle of altitude $\theta$ (see Fig. 3) and the effects of the cables aerodynamic drag as in [12] and [29], respectively. In short, the basic assumptions are:

- flight at constant altitude $\theta$ and zero azimuth angle with respect to the wind direction ${ }^{1}$;

- negligible inertia and gravity forces of the aircraft with respect to the aerodynamic loads;

- high equivalent aerodynamic efficiency;

- therefore, steady state flight in crosswind direction is assumed.

These approximations are typically adopted in the literature of AWECs and are commonly used for first design iterations, even though experimental validations are challenging and the results are scattered [30]. This first model allows fast analytical computations and is therefore very useful for our preliminary assessment of offshore AWECs.

\footnotetext{
${ }^{1}$ Notice that this last hypothesis defines a theoretical horizontal flight which describes only the instant of time when the aircraft crosses the zero azimuth. In order to imagine an equivalent more realistic flight path that satisfies this requirement, one should think to a semicircular flight path around the wind direction such that the angular distance of the aircraft from the wind direction is kept constant. A good definition of such distance is given in [13].
} 


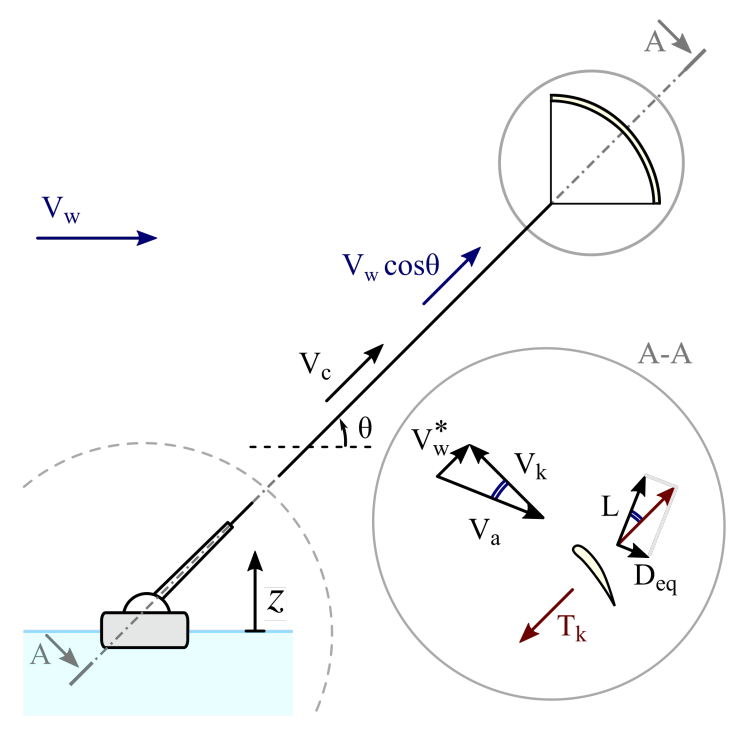

Figure 3: AWEC aerodynamic model - The balloon shows the aerodynamic equilibrium at the kite in the tether reference system.

With reference to Fig. 3, it is possible to derive the equations that govern the aircraft dynamics.

In particular, assuming the following notation: $V_{\mathrm{k}}$ is the absolute aircraft speed, $V_{\mathrm{a}}$ is the apparent wind speed, $V_{\mathrm{w}}$ is the actual wind speed, $V_{\mathrm{c}}$ is the velocity of the cable in the direction its own axis, $T_{\mathrm{k}}$ is the tether traction force, $L$ is the aircraft lift force, $D_{\text {eq }}$ is the equivalent drag force (i.e the drag force of the aircraft plus the equivalent cable drag force acting on the aircraft), and $V_{\mathrm{w}}^{*}$ is the wind speed felt by the aircraft defined as

$$
V_{\mathrm{w}}^{*}=V_{\mathrm{w}} \cos \theta-V_{\mathrm{c}}
$$

Notice that, with reference to Fig. 3, the force equilibrium at the aircraft makes the velocity triangle and the force triangle similar, thus yielding

$$
V_{\mathrm{k}}=E_{\mathrm{eq}} V_{\mathrm{w}}^{*}
$$

The equivalent aerodynamic efficiency takes account of the cables aerodynamic drag and can be derived by computing the energy dissipated by the 
distributed cable drag and reads as

$$
E_{\text {eq }}=\frac{C_{\mathrm{L}}}{C_{\mathrm{D}}+\frac{C_{\perp} n_{\mathrm{c}} r_{\mathrm{c}} d_{\mathrm{c}}}{4 A}}=\frac{L}{D_{\mathrm{eq}}}
$$

$$
\begin{gathered}
\left(M+M_{\infty}+M_{\mathrm{m}}\right) \ddot{z}+\int_{0}^{t} k_{\mathrm{r}}(t-\tau) \dot{z}(\tau) \mathrm{d} \tau+B_{\mathrm{m}} \dot{z}+\left(k_{\mathrm{b}}+k_{\mathrm{m}}\right) z \\
=\sum_{\mathrm{i}=1}^{n} F_{\mathrm{wi}} \sin \left(\omega_{\mathrm{i}} t+\phi_{\mathrm{i}}\right)+T_{\mathrm{k}} \sin \theta \\
V_{\mathrm{r}}=V_{\mathrm{w}} \cos \theta-\dot{z} \sin \theta-\sqrt{\frac{T_{\mathrm{k}}}{\frac{1}{2} \rho_{\mathrm{a}} E_{\mathrm{eq}}^{2} C_{\mathrm{L}} A}} \\
P=T_{\mathrm{k}} V_{\mathrm{r}}
\end{gathered}
$$


Eq. 10 is the platform equation that defines the vertical motion of the floating structure. Eq. 11 is the winch equation that couples the platform dynamics with the kite model and defines the cables reel out velocity as seen by the winches. It is given by the combination of equations 5,8 and 9 . Eq. 12 is the power equation that defines the instantaneous available power to the alternators, $P$ as the product between the tether tension, $T_{\mathrm{k}}$, and the cables reel-out velocity as seen by a reference system that is fixed on the platform, $V_{\mathrm{r}}$.

\subsection{Control}

The tether force, $T_{\mathrm{k}}$, can be controlled thanks to the drums reeling velocity $V_{\mathrm{r}}$. For example, if the cables are reeling-in, the wind speed felt by the aircraft $V_{\mathrm{w}}^{*}$ increases, thus increasing the flight speed and the aerodynamic lift. It is easy to understand that a controller can decouple the buoy from the aircraft by imposing an appropriate velocity to the drums in order to cancel the effect of the buoy motion on the cable speed $V_{\mathrm{c}}$.

However, it also is possible to envisage a more complex controller that makes it possible to harvest energy, not only from wind, but also from waves without

230 any changes in system architecture. Specifically, a suitable control on the force $T_{\mathrm{k}}$ can be conceived in order to exert an oscillating force on the platform and to extract energy from waves by damping its heaving motion. In order to assess this potential improvement in the power output, the average combined wind-wave power output of the floating AWEC has to be computed. In the following, before analysing the case of combined wind-wave power, the formulations for estimating the maximum wind-only and wave-only power extraction are provided.

\subsubsection{Wind-only optimal power output}

For the well known case of a ground based pumping AWEC, Eqs. 11 and 12 can be simplified by fixing the heave coordinate, $\mathrm{z}(\mathrm{t})=0$.

The optimal reel out speed is known to be $V_{\mathrm{r} 0}=1 / 3 V_{\mathrm{w}} \cos \theta$ [28]. In such a case, the optimal aircraft tether force of Eq. 8 is constant:

$$
T_{\mathrm{k}}=T_{\mathrm{k} 0}
$$



power, $P_{0}=1 / 2 \rho_{\mathrm{a}} V_{\mathrm{w}}^{3} 4 / 27 E_{\mathrm{eq}}^{2} C_{\mathrm{L}} A \cos ^{3} \theta$.

\subsubsection{Wave-only optimal power output}

Eq. 10 is the equation of motion of a generic moored WEC, where $T_{\mathrm{k}} \sin \theta$ corresponds to a general force of an external Power Take Off (PTO) unit, that

can be externally controlled to introduce an additional mechanical impedance on the platform heaving motion and thereby extracting power from the waves.

Assuming regular waves, as typically made in preliminary analyses of WEC concepts [31], the wave force on the platform reads as $f_{\mathrm{e}}=F_{\mathrm{w}} \sin (\omega t)$ and then the integral term of Eq. 10 can be simplified in

$$
\int_{0}^{t} k_{\mathrm{r}}(t-\tau) \dot{z}(\tau) \mathrm{d} \tau=\left(M_{\text {add }}(\omega)-M_{\infty}\right) \ddot{z}(t)+B_{\mathrm{r}}(\omega) \dot{z}(t)
$$

250 AWEC hydrodynamic parameters [32].

\subsection{Combined wind-wave power output}

In order to investigate the possibility of extracting combined wind-wave power output, the tether force is then assumed to be a combination of a constant

The controller equation assumes the following form

$$
T_{\mathrm{k}}=\mathrm{c} T_{\mathrm{k} 0}-r_{\mathrm{g}} \dot{z}(t)-s_{\mathrm{g}} z(t)
$$


This control strategy introduces a superimposed alternate motion of the cables, due to the intrinsic relation between traction force and kite velocity (given by Eq. 8). This means that the aerodynamically-optimal reel out speed (given in [28]) cannot be followed. Therefore, the introduction of such a controller is reducing the amount of extracted wind power with respect to the ideal maximum. In order to evaluate if the global balance is positive, having the benefit from the additional power from waves overcoming the losses from wind, the global wind-wave power output of the floating AWEC has to be computed. Using Eqs. 10, 11, 12 and 14, simplifying the convolution integral with Eq. 13 and integrating on the wave period $T_{\mathrm{w}}$, it is possible to derive the analytical expression for the steady state average power output of the combined wind-wave generation, $P_{\mathrm{ww}}$.

$$
\begin{array}{r}
P_{\mathrm{ww}}=c T_{\mathrm{k} 0} V_{\mathrm{w}} \cos \theta-\frac{1}{T_{\mathrm{w}}} \int_{0}^{T_{\mathrm{w}}} c T_{\mathrm{k} 0} C(t) \mathrm{d} t+\frac{1}{2} z_{1}^{2} \omega^{2} r_{\mathrm{g}} \sin \theta \\
+\frac{1}{T_{\mathrm{w}}} \int_{0}^{T_{\mathrm{w}}} \dot{z}(t) r_{\mathrm{g}} C(t) \mathrm{d} t+\frac{1}{T_{\mathrm{w}}} \int_{0}^{T_{\mathrm{w}}} z(t) s_{\mathrm{g}} C(t) \mathrm{d} t
\end{array}
$$

with

$$
C(t)=\sqrt{\frac{c T_{\mathrm{k} 0}-r_{\mathrm{g}} \dot{z}(t)-s_{\mathrm{g}} z(t)}{\frac{1}{2} \rho_{\mathrm{a}} E_{\mathrm{eq}}^{2} C_{\mathrm{L}} A}}
$$

The solution for the dynamics of the platform reads as

$$
\begin{array}{r}
z(t)=z_{1} \sin \left(\omega t+\phi_{\mathrm{z}}\right) \\
\dot{z}(t)=\omega z_{1} \cos \left(\omega t+\phi_{\mathrm{z}}\right)
\end{array}
$$

with

$$
\begin{gathered}
z_{1}=\frac{F_{\mathrm{w}}}{\sqrt{\left(k-\omega^{2} m\right)^{2}+\omega^{2} r^{2}}} \\
\phi_{\mathrm{z}}=-\arctan \left(\frac{\omega r}{k-\omega^{2} m}\right)
\end{gathered}
$$




$$
\begin{array}{r}
m=M+M_{\text {add }}(\omega)+M_{\mathrm{m}} \\
r=B_{\mathrm{r}}+B_{\mathrm{m}}+r_{\mathrm{g}} \sin \theta \\
k=k_{\mathrm{b}}+k_{\mathrm{m}}+s_{\mathrm{g}} \sin \theta
\end{array}
$$

If $r_{\mathrm{g}}$ and $s_{\mathrm{g}}$ are zero, then the maximum $P_{\mathrm{ww}}$ is achieved with $c=1$ and easily guess, that an AWEC placed on a floating platform is capable of generating at least the same power as if it were fixed on the ground exposed to the same absolute wind. However, Eq. 15 can be numerically maximised in order to find the optimal values of the controller parameters and verify if it is possible

\section{Case study}

In this section, the model provided in section 3 is implemented in a numerical case study.

\subsection{Geometry}

The geometry of the platform is assumed to be of cylindrical shape and to be moored with a single line catenary mooring. A wide range of geometries and sea states have been investigated, but we report only the few most significant results whose details are provided in Fig. 4 and Tables 1, 2, 3.

\subsection{Computation of the hydrodynamic coefficients}

295

For all the considered cases, the hydrodynamic coefficients $M_{\infty}, M_{\text {add }}, B_{\mathrm{r}}$, $F_{\mathrm{w}}, M_{\mathrm{m}}, B_{\mathrm{m}}, k_{\mathrm{m}}$ were computed for the chosen geometries with a standard linear radiation-diffraction software (WAMIT). The mooring coefficients were taken from [27] and dimensioned in such a way to balance the horizontal forces of the kite cable.

The convolution integral found in Eq. 10 can be solved in a computationally efficient manner by approximating it with a system of differential equations. Sufficient accuracy can be achieved with a system of the third order or 

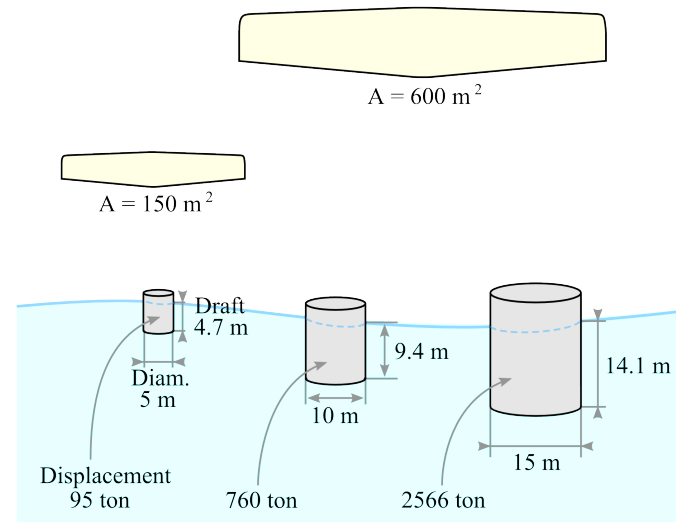

Figure 4: Platform and aircraft dimensions - The picture shows the dimensions of platforms and aircraft properly scaled. The draft is the submerged height and is chosen to be equal to 1.88 times the radius.

\begin{tabular}{llll}
\hline \hline Aircraft Data & & & Unit \\
\hline \hline Aerodynamic efficiency $E_{\text {eq }}$ & 10 & & \\
Lift coefficient $C_{\mathrm{L}}$ & 0.65 & & \\
Air density $\rho_{\mathrm{a}}$ & 1.225 & & $\mathrm{~kg} / \mathrm{m}^{3}$ \\
Flight elevation angle $\theta$ & 45 & & $\mathrm{deg}$ \\
Wind speed $V_{\mathrm{w}}$ & 12 & & $\mathrm{~m} / \mathrm{s}$ \\
\hline & Small & $\mathrm{Big}$ & \\
\hline Area $A$ & 150 & 600 & $\mathrm{~m}^{2}$ \\
Nominal tension $T_{\mathrm{k} 0}$ & 191 & 764 & $\mathrm{kN}$ \\
Nominal power $P_{0}$ & 0.54 & 2.16 & $\mathrm{MW}$ \\
\hline
\end{tabular}

Table 1: Aircraft data. 


\begin{tabular}{lllll}
\hline \hline Platform Data & Small & Medium Big & Unit \\
\hline \hline Diameter $D_{\mathrm{b}}$ & 5 & 10 & 15 & $\mathrm{~m}$ \\
Draft & 4.7 & 9.4 & 14.1 & $\mathrm{~m}$ \\
Nominal displacement & 95 & 760 & 2566 & ton \\
Added mass $M_{\infty}$ & 31 & 249 & 842 & ton \\
Buoyancy stiffness $k_{\mathrm{b}}$ & 198 & 793 & 1784 & $\mathrm{kN} / \mathrm{m}$ \\
Natural period $($ ca. $)$ & 5.2 & 7.1 & 8.6 & $\mathrm{sec}$ \\
\hline
\end{tabular}

Table 2: Platform data.

\begin{tabular}{llll}
\hline \hline Mooring Data & & & Unit \\
\hline \hline Water depth & 50 & & $\mathrm{~m}$ \\
Length & 350 & & $\mathrm{~m}$ \\
Chain diameter & 64 & & $\mathrm{~mm}$ \\
Linear mass in air & 90 & & $\mathrm{~kg} / \mathrm{m}$ \\
\hline & Small & Big & \\
& air- & air- & \\
& craft & craft & \\
\hline Equivalent mass $M_{\mathrm{m}}$ & 33.1 & 53.2 & $\mathrm{ton}$ \\
Equivalent damping $B_{\mathrm{m}}$ & 17954 & 35084 & $\mathrm{~N} /(\mathrm{m} / \mathrm{s})$ \\
Equivalent stiffness $k_{\mathrm{m}}$ & 5940 & 11331 & $\mathrm{~N} / \mathrm{m}$ \\
\hline
\end{tabular}

Table 3: Single line inelastic catenary mooring data. The equivalent mechanical properties change significantly with the loading condition. 
higher [33]. In this case, a fourth order linear system is used and its coefficients were computed by using $M_{\infty}, M_{\text {add }}(\omega)$ and $B_{\mathrm{r}}(\omega)$.

The static buoyancy stiffness $k_{\mathrm{b}}$ can be easily computed as the product of water density, gravity acceleration, and waterplane area $\rho_{\mathrm{w}} g \pi D_{\mathrm{b}}^{2} / 4$, where $D_{\mathrm{b}}$ is the platform diameter.

\subsection{Results}

A numerical parametric study of Eq. 15 in eight different sea states was performed for each geometry. The goal was to maximize the power output $P_{\mathrm{ww}}$ and the results are shown in Fig. 5.

In Fig. 5 each coloured square has two numbers. The first number (without brackets) is the wave power potential improvement and is the ratio $P_{\mathrm{w}} / P_{0}$ where $P_{\mathrm{w}}$ is the nominal wave power that reaches the floating platform computed by multiplying the linear wave power density ${ }^{2}, P_{\mathrm{wl}}$, by the platform diameter $D_{\mathrm{b}}$.

The second number (within brackets) is the wind-wave power actual improvement and is computed as $\left(P_{\mathrm{ww}}-P_{0}\right) / P_{0}$. Finally the colormap of the cells represents how much of the wave potential is exploited (in addition to the wind potential); it is given by the ratio between the second and the first number and 320 is then $\left(P_{\mathrm{ww}}-P_{0}\right) / P_{\mathrm{w}}$.

The picture also shows the values of the buoy resonance period $T_{\mathrm{b}}=\frac{2 \pi}{\sqrt{k / m}}$ and the lift safety $\eta$. The lift safety is the nominal mass of the platform plus the mooring line, divided by the maximum steady state aircraft tension. If an offshore wind-only AWEC is to be built, it is reasonable to expect $\eta>1.5$.

325 4.4. Small aircraft - Small platform

In Fig. $5 \mathrm{~A}$, the platform is well dimensioned ( $\eta$ around 5 or 6 ), meaning that more than 5 times the nominal aircraft pull is needed to lift the whole system out of the water. The potential wave power advantage ranges anywhere

\footnotetext{
${ }^{2}$ The linear wave power density $(\mathrm{W} / \mathrm{m})$ in deep water can be computed with the formula $P_{\mathrm{wl}}=\frac{\rho_{\mathrm{w}} g^{2} T_{\mathrm{w}} H_{\mathrm{w}}^{2}}{32 \pi}$ where $H_{\mathrm{w}}$ is the wave peak-to-peak amplitude.
} 


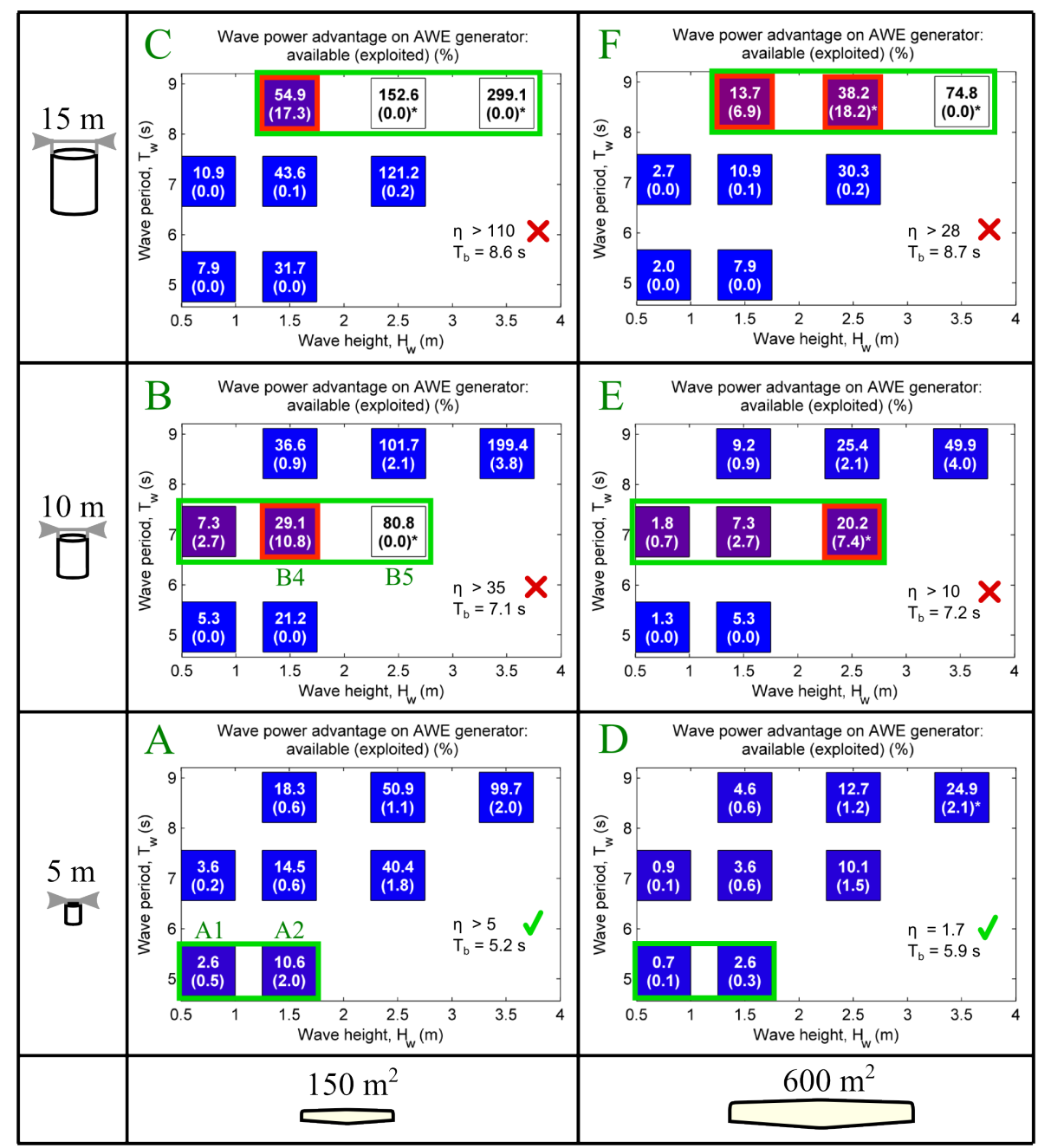

Figure 5: Potential wave power advantage on different offshore geometries - Each coloured square shows two numbers: the first (without brackets) is the incident wave power on the hull of the AWEC as a percentage of the nominal wind-only power, the second (within brackets) is the actual improvement on the power output that is obtained with wind-wave generation instead of wind-only generation. The background colour of the cell represents the ratio between the second and the first number thus giving a visual representation of the ability to exploit the potential advantage. The cells with actual improvement higher than $5 \%$ are marked in red. Resonant sea states are marked in green. An ' $\mathrm{x}$ ' or a check mark indicates whether the platform is oversized or not with respect to a wind-only generator. Systems with not oversized platforms experience the lowest performance improvements. 
between $2.6 \%$ and $99.7 \%$ However, the improvement that is actually achieved by configuration $\mathrm{A}$ is at most $2.0 \%$ even in the resonant sea states (inside the green box) where $T_{\mathrm{w}}$ is roughly equal to $T_{\mathrm{b}}$.

\subsection{Small aircraft - Medium platform}

If the buoy size is increased, Fig. 5B, $\eta$ increases, meaning that the buoy is heavy when compared to the aircraft force. If compared to case $\mathrm{A}$, the platform resonates with sea states that carry a larger amount of wave power (potential advantage up to $80 \%$ in B5), however the best power output is achieved in B4 (aircraft-buoy combination B, sea state n. 4) where the improvement is only $10.8 \%$ with respect to wind-only generation. Sea state data for case A2 and B4 are shown in Table 4.

Sea state B5 is marked by a ${ }^{*}$ and is white. It has a $*$ because the platform oscillations are large comparing to its own dimensions thus reducing the results' reliability. When such oscillations are considered too large, the cell is also whitefilled and the advantage is set to zero $\left(s_{\mathrm{g}}=r_{\mathrm{g}}=0\right)$. In this case the aircraft can be controlled at constant nominal tension yielding the wind-only power ${ }_{345} P_{\mathrm{ww}}=P_{0}$.

\subsection{Small aircraft - Big platform}

If the buoy is enlarged even more, Fig. 5C, the advantage increases to $17.3 \%$ at the cost of having a huge platform with respect to the wind-only needs $(\eta>110)$.

\subsection{Big aircraft}

Increasing the aircraft size does not change the results discussed for the small aircraft. Since the aircraft area increased by a factor 4 , the potential advantages are much smaller $(1 / 4)$ than for the small aircraft. Moreover, in case $\mathrm{D}$ the platform could be lifted out of water by peak forces. 


\subsection{Discussion}

The fact that WECs performance are optimal only when they resonate with the sea state explains why all the cells outside the green boxes (i.e. non resonating working conditions) are coloured in blue (i.e. the obtained improvement is low). However, Fig. 5 clearly shows that the potential advantage of having a combined wind-wave system instead of a wind-only generator does not provide major benefits in most of the circumstances, even inside the green boxes. This can be explained as follows. It is possible to see from Eqs. 5, 9, 10, 11 and 14 that in order to extract energy from the wave, $r_{\mathrm{g}}$ must be greater than zero, thus requiring $T_{\mathrm{k}}$ to be different from the nominal $T_{\mathrm{k} 0}$. The wind speed at the aircraft, $V_{\mathrm{w}}^{*}$, is therefore different from the optimal $\left(2 / 3 V_{\mathrm{w}} \cos \theta\right)$ violating the aerodynamic optimum. This can be seen in Fig. 6 where case B4 is analysed. The average power output, $P_{\mathrm{ww}}$, (top) as a function of $r_{\mathrm{g}}$ is obtained from Eq. 15 by fixing the numerically optimal $s_{\mathrm{g}}$ and $c$. It is worth noticing that the optimal value of $c$ is very close to one for all the considered cases. The average power contribution due to the wave (bottom) is computed as $\frac{1}{T_{\mathrm{w}}} \int_{0}^{T_{\mathrm{w}}} \dot{z}(t)^{2} r_{\mathrm{g}} \sin \theta \mathrm{d} t=\frac{1}{2}\left(\omega z_{1}\right)^{2} r_{\mathrm{g}} \sin \theta$. The average wind power (middle) is computed as the difference between the other two.

\begin{tabular}{llll}
\hline \hline Sea Data & & & Unit \\
\hline \hline Water density $\rho_{\mathrm{w}}$ & 1030 & & $\mathrm{~kg} / \mathrm{m}^{3}$ \\
\hline Sea State & $\mathrm{n} .2$ & $\mathrm{n} .4$ & \\
\hline Frequency $\omega$ & 1.21 & 0.89 & $\mathrm{rad} / \mathrm{s}$ \\
Period $T_{\mathrm{w}}$ & 5.16 & 7.06 & $\mathrm{sec}$ \\
Peak-to-peak height $H_{\mathrm{w}}$ & 1.5 & 2.5 & $\mathrm{~m}$ \\
Power density $P_{\mathrm{wl}}$ & 11.4 & 43.6 & $\mathrm{~kW} / \mathrm{m}$ \\
\hline
\end{tabular}

Table 4: Wave data used to simulate cases A2 (n. 2) and B4 (n. 4).

\subsection{Transient behaviour}

So far, only the steady state values that occur during a continuous reel- 

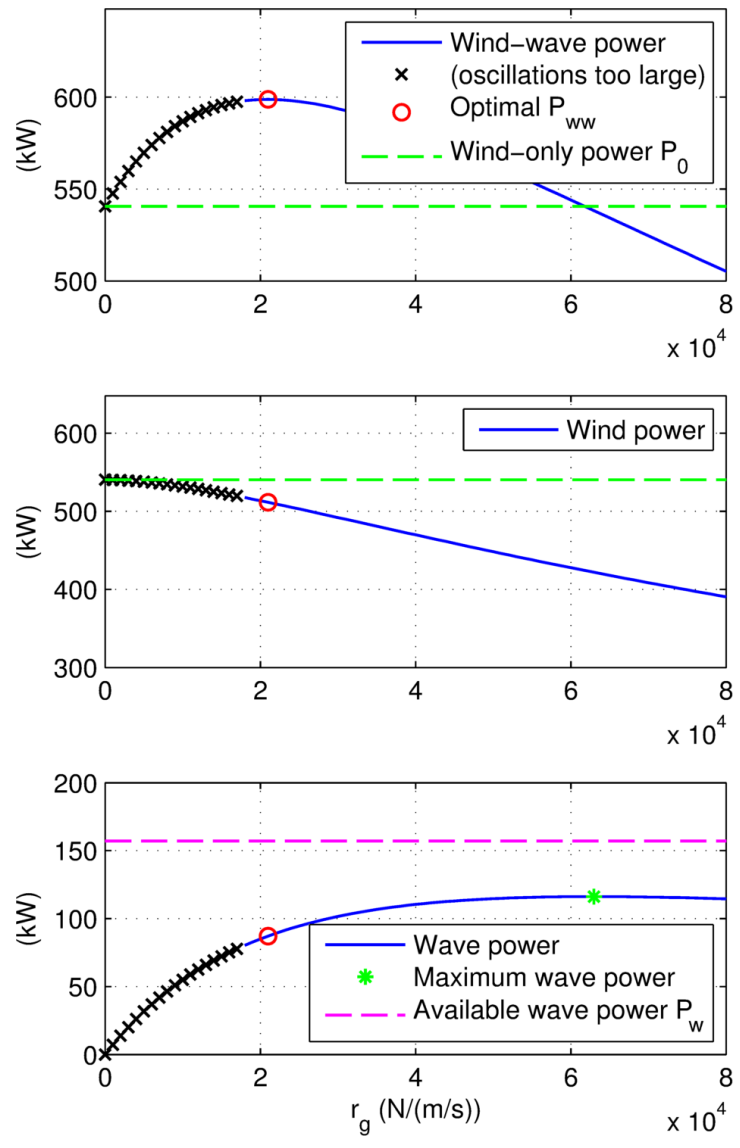

Figure 6: Combined wind-wave power output, case B4 of fig. 5 - Power output (top), wind power (middle) and wave power (bottom) as a function of $r_{\mathrm{g}}$. The top graph is the sum of the other two. The dashed lines represent the nominal wind-only and wave-only power, $P_{0}$ and $P_{\mathrm{w}}$ respectively. 
floating platform (case A2 of fig. 5) in case the pumping cycle is performed. The equations of motion are the same of section 3.3 with the only difference that the tether force is multiplied by a square wave $q(t)$ having unit amplitude and $60 \%$ reel-out time. $T_{\mathrm{k}}$ is then substituted with $T_{\mathrm{k} d}=q(t) T_{\mathrm{k}}$. According to this hypothesis the transient motion has a negligible impact on the average power output that results equal to $61.4 \%$ of $P_{0}(2.4 \%$ higher than the nominal $60 \%$ of $\left.P_{0}\right)$.
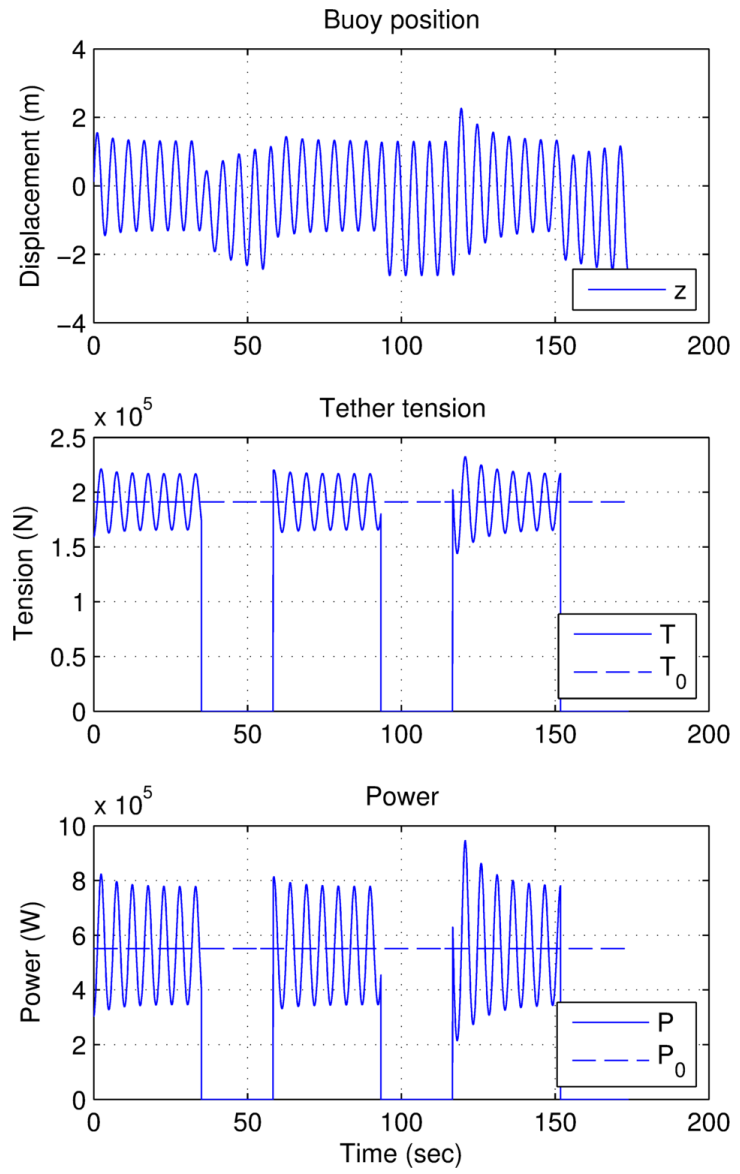

Figure 7: Offshore AWEC transient behaviour. - Typical simulated heave motion of the platform (top), aircraft tether force (middle) and mechanical power output (bottom). Three pumping cycles are shown. Even though the platform reaches steady state conditions in about $30 \mathrm{sec}$, the three cycles are different because the duty cycle period is not a multiple of the wave period. 


\subsection{Applicability of the results}

The results shown in section 4 represent the first parametric analysis of the

385

and models that have been adopted were partly taken from the literature of other engineering fields such as ocean wave and Airborne Wind Energy. As for the hydrodynamics, the buoy was modelled as a heaving rigid body with only one DoF whereas other translations and all the rotations have been completely neglected. Among them, the surge and pitch motions could be also relevant since traction force of the aircraft may have relevant components along these two directions, depending on the structural layout. Moreover, as discussed in section 3.1.1, the structural forces are computed thanks to linear wave theory and potential flow hydrodynamics, thus limiting the applicability of the results only to non-extreme operational conditions. As for the aerodynamics, the simple steady state model introduced in section 3.2 aims at representing the aircraft behaviour without considering several aspects of the dynamics of a real airborne system such as the effects of gravity (weight of the aircraft), wind gusts, deformation of the wing, changes in lift or drag coefficients, cable vibration/galloping or other aeroelastic fluttering etc. However, the approximations used in this study are very helpful for an initial analysis, allowing to introduce for the first time the offshore floating AWEC concept and to provide a preliminary assessment of its performance.

\section{Conclusions}

The dynamics of an offshore Airborne Wind Energy Converter (AWEC) were investigated by coupling the aircraft steady state crosswind model with the 1 degree of freedom hydrodynamics of a floating platform held in place by a catenary mooring line. A simple analytical model to compute the power production and the platform heaving motion was derived. The model shows clearly that offshore AWECs are viable and also that there is a mild potential improvement due to combined wind-wave energy exploitation that can be achieved without changing 
the generator design. The model was numerically optimized and simulated with six different combinations of aircraft-platform sizes in eight different sea states. Despite this simple and fast model allows first design iterations, further research is required in modelling the system hydrodynamics in order to take account of other important factors such as heave-pitch-surge motion and response in irregular sea. As regards the shape of the platform, other geometries could be considered in future works with the aim of improving the performances of the combined wind-wave power extraction.

Moreover, the proposed analytical tools should be extended to the modeling of AWEC farms which are expected to be much more efficient from the technoeconomic point of view.

\section{Acknowledgements}

This work was carried out with the financial support of Kitegen Research Srl and Scuola Superiore Sant'Anna. David Forehand from Edinburgh University provided the hydrodynamic coefficients. The authors would also like to thank Mr. Giacomo Moretti and Mr. Fabio Calamita for their help.

\section{References}

[1] A. Cherubini, A. Papini, R. Vertechy, M. Fontana, Airborne wind energy systems: A review of the technologies, Renewable and Sustainable Energy Reviews 51 (2015) 1461-1476. doi:10.1016/j.rser.2015.07.053.

[2] C. L. Archer, An introduction to meteorology for airborne wind energy, Airborne Wind Energy, U. Ahrens, M. Diehl, R. Schmehl Eds., Springer Berlin, Chapter 5, p 81 - 94, 2013.

435

[3] M. Diehl, Airborne wind energy: Basic concepts and physical foundations, Airborne Wind Energy, U. Ahrens, M. Diehl, R. Schmehl Eds., Springer Berlin, Chapter 1, p 3 - 22, 2013. 
[4] M. Ippolito, Wind energy conversion system with kites towing modules on a rail, PCT patent application, WO2014087436, 2014.

[5] EWEA, European wind energy association: Wind in power 2013, european statistics, http://www.ewea.org/fileadmin/files/library/publications/ statistics/EWEA_Annual_Statistics_2013.pdf , 2014.

[6] M. Bilgili, A. Yasar, E. Simsek, Offshore wind power development in europe and its comparison with onshore counterpart, Renewable and Sustainable Energy Reviews 15 (2) (2011) 905 - 915.

[7] NREL, National renewable energy laboratory: Large-scale offshore wind power in the united states, assessment of opportunities and barriers, http://www.nrel.gov/docs/fy10osti/40745.pdf , 2010.

[8] B. Skaare, T. D. Hanson, R. Yttervik, F. G. Nielsen, Dynamic response and control of the hywind demo floating wind turbine, Proceedings of EWEA Conference, 2011.

[9] M. Erhard, H. Strauch, Flight control of tethered kites and winch control for autonomous airborne wind energy generation in pumping cycles, Control Engineering Practice 40 (2015) 13-26.

[10] U. Fechner, R. Schmehl, Model-based efficiency analysis of wind power conversion by a pumping kite power system, Airborne Wind Energy, U. Ahrens, M. Diehl, R. Schmehl Eds., Springer Berlin, Chapter 14, p 249 $269,2013$.

[11] G. Horn, S. Gros, M. Diehl, Numerical trajectory optimization for airborne wind energy systems described by high fidelity aircraft models, Airborne Wind Energy, U. Ahrens, M. Diehl, R. Schmehl Eds., Springer Berlin, Chapter 11, p 205 - 218, 2013.

[12] I. Argatov, P. Rautakorpi, R. Silvennoinen, Estimation of the mechanical energy output of the kite wind generator, Renewable Energy 34 (6) (2009) $1525-1532$. 
[13] M. Erhard, H. Strauch, Theory and experimental validation of a simple comprehensible model of tethered kite dynamics used for controller design, Airborne Wind Energy, U. Ahrens, M. Diehl, R. Schmehl Eds., Springer Berlin, Chapter 8, p 141 - 165, 2013.

[14] J. Breukels, R. Schmehl, W. Ockels, Aeroelastic simulation of flexible membrane wings based on multibody system dynamics, Airborne Wind Energy, U. Ahrens, M. Diehl, R. Schmehl Eds., Springer Berlin, Chapter 16, p 287 - 305, 2013.

[15] P. Williams, B. Lansdorp, W. Ockels, Modeling and control of a kite on a variable length flexible inelastic tether, AIAA Modelling and Simulation Technologies Conference and Exhibit, Hilton Head, SC, USA (2007) 6705.

[16] I. Argatov, P. Rautakorpi, R. Silvennoinen, Apparent wind load effects on the tether of a kite power generator, Journal of Wind Engineering and Industrial Aerodynamics 99 (10) (2011) 1079 - 1088.

[17] O. M. Faltinsen, Sea loads on ships and offshore structures, Cambridge University Press, Cambridge, 1990.

[18] A. F. de O. Falco, Wave energy utilization: A review of the technologies, Renewable and Sustainable Energy Reviews 14 (3) (2010) 899 - 918.

[19] A. Babarit, J. Hals, M. Muliawan, A. Kurniawan, T. Moan, J. Krokstad, Numerical benchmarking study of a selection of wave energy converters, Renewable Energy 41 (0) (2012) $44-63$.

[20] R. A. Skop, Mooring systems: A state-of-the-art review, J. Offshore Mech. Arct. Eng. 110 (4) (1988) $365-372$.

[21] R. E. Harris, L. Johanning, J. Wolfram, Mooring systems for wave energy converters: A review of design issues and choices, Paper presented at The Institute of Marine Engineering, Science and Technology (IMarEST) (2004) $180-189$. 
[22] L. Johanning, G. H. Smith, J. Wolfram, Mooring design approach for wave energy converters, Proceedings of the Institution of Mechanical Engineers. Part M, Journal of engineering for the maritime environment (online) 220 (4) (2006) $159-174$.

[23] M. E. McCormick, Ocean wave energy conversion, Dover publications Inc., Mineola, New York, 2007.

[24] T. Soulard, M. Alves, A. Sarmento, Force reacting principle applied to a heave point absorber wave energy converter, Proceedings of the International Offshore and Polar Engineering Conference (2009) 312-318.

[25] J. Falnes, on non-causal impulse response functions related to propagating water waves, Applied Ocean Research 17 (6) (1995) 379 - 389.

[26] M. Alves, Numerical simulation of the dynamics of point absorber wave energy converters using frequency and time domain approaches, $\mathrm{PhD}$ Thesis, 2013.

[27] F. Cerveira, N. Fonseca, R. Pascoal, Mooring system influence on the efficiency of wave energy converters, International Journal of Marine Energy 34 (0) (2013) $65-81$.

[28] M. L. Loyd, Crosswind kite power (for large-scale wind power production), Journal of Energy 4 (3) (1980) 106 - 111.

[29] B. Houska, M. Diehl, Optimal control of towing kites, Proceedings of the 45th IEEE conference on decision \& control, San Diego, USA (2006) 2693 $-2697$.

[30] D. V. Lind, Analysis and Flight Test Validation of High Performance Airborne Wind Turbines, Airborne Wind Energy, U. Ahrens, M. Diehl, R. Schmehl Eds. (Springer Berlin), Chapter 28, 473 - 490, (2013).

[31] G. Moretti, D. Forehand, R. Vertechy, M. Fontana, D. Ingram, Modeling of an oscillating wave surge converter with dielectric elastomer power take- 
off, ASME 33rd International Conference on Ocean, Offshore and Arctic Engineering (2014).

[32] J. Falnes, Ocean waves and oscillating systems, Cambridge University Press, Cambridge, 2004.

[33] Z. Yu, J. Falnes, state-space modelling of a vertical cylinder in heave, Applied Ocean Research 17 (5) (1995) 265 - 275. 